\title{
Variáveis que influenciam a manutenção do aleitamento materno exclusivo
}

\author{
VARIABLES THAT INFLUENCE THE MAINTENANCE OF EXCLUSIVE BREASTFEEDING \\ VARIABLES QUE INFLUENCIAN LA MANUTENCIÓN DEL AMAMANTAMIENTO MATERNO \\ EXCLUSIVO
}

\author{
Nádia Zanon Narchi', Rosa Áurea Quintella Fernandes², Lílian de Araújo Dias ${ }^{3}$, \\ Daniela Higasa Novais ${ }^{4}$
}

\begin{abstract}
RESUMO
Pesquisa descritiva, exploratória e retrospectiva, com abordagem quantitativa, realizada em uma comunidade carente de São Paulo com o objetivo de verificar se a manutenção do aleitamento exclusivo (AE) nos primeiros seis meses é influenciada pelas variáveis: contato precoce na primeira hora após o nascimento, permanência em alojamento conjunto, tipo de parto e tipo de hospital. Os dados foram coletados de 75 prontuários e analisados com a metodologia de equações de estimação generalizada. Os resultados mostraram que as variáveis alojamento conjunto, tipo de hospital e tipo de parto interferiram na manutenção do $A E$, o mesmo não ocorrendo com o contato precoce. Concluiu-se que os índices de $A E$ foram maiores nos casos em que mãe e bebê permaneceram constantemente juntos após o parto, em hospitais amigos da criança e após partos normais. Constatou-se que a assistência recebida pela mulher durante o processo de parto e nascimento influencia de forma direta a amamentação.
\end{abstract}

\section{DESCRITORES}

Aleitamento materno.

Promoção da saúde.

Saúde materno-infantil.

\begin{abstract}
This is a descriptive, exploratory and retrospective study, with a quantitative approach, performed in a low-income community in São Paulo, with the purpose to identify whether the maintenance of exclusive breastfeeding (EBF) in the first six months is influenced by the following variables: early contact in the first hour after birth, permanence in joint lodging, type of delivery and type of hospital. Data were collected from 75 medical records and analyzed with the methodology of generalized estimate equations. The results showed that the variables joint lodging, type of hospital and type of delivery interfered in the maintenance of EBF; however, that was not the case with early contact. It was concluded that the EBF indexes were higher in cases where the mother and the baby remained together after the birth, in babyfriendly hospitals and after normal deliveries. It was also observed that the care received by the mother during the process of delivery and birth influences breastfeeding directly.
\end{abstract}

\author{
KEY WORDS \\ Breast feeding. \\ Health promotion. \\ Maternal and child health.
}

\begin{abstract}
RESUMEN
Se trata de una investigación descriptiva, exploratoria y retrospectiva, con abordaje cuantitativo, realizada en una comunidad carente de San Pablo, con el objetivo de verificar si la manutención del amamantar exclusivo ( $A E$ ) en los primeros seis meses fue influenciada por las variables: contacto precoz en la primera hora después del nacimiento, permanencia en el mismo alojamiento, tipo de parto y tipo de hospital. Los datos fueron recolectados de 75 registros y analizados con la metodología de ecuaciones de cálculo generalizado. Los resultados mostraron que las variables alojamiento conjunto, tipo de hospital y tipo de parto interfirieron en la manutención del $A E$, lo mismo no ocurrió con el contacto precoz. Se concluye que los índices de AE fueron mayores en los casos en que la madre $y$ el bebé permanecieron constantemente juntos después del parto, en hospitales amigos del niño y después de partos normales. Se constató que la asistencia recibida por la mujer durante el proceso de parto y nacimiento influye de forma directa en el amamantar.
\end{abstract}

\section{DESCRIPTORES}

Lactancia materna.

Promoción de la salud.

Salud materno-infantil.

${ }^{1}$ Enfermeira Obstétrica. Doutora em Enfermagem. Docente do Curso de Obstetrícia da Escola de Artes, Ciências e Humanidades da Universidade de São Paulo. Participante do Grupo de Pesquisa: O cuidar em enfermagem na saúde da mulher, da criança e do adolescente. São Paulo, SP, Brasil. nzn@usp.br. ${ }^{2}$ Obstetriz. Doutora em Enfermagem. Docente do Curso de Mestrado em Enfermagem da Universidade Guarulhos. Líder do Grupo de Pesquisa: O cuidar em enfermagem na saúde da mulher, da criança e do adolescente. São Paulo, SP, Brasil. fernands@uol.com.br. ${ }^{3}$ Enfermeira. Participante do Grupo de Pesquisa: O cuidar em enfermagem na saúde da mulher, da criança e do adolescente. Guarulhos, SP, Brasil. lilinharosa21@yahoo.com.br. ${ }^{4}$ Enfermeira. Aluna do Curso de Especialização em Enfermagem Obstétrica da Universidade Federal de São Paulo. Participante do Grupo de Pesquisa: O cuidar em enfermagem na saúde da mulher, da criança e do adolescente. São Paulo, SP, Brasil. danielanovais@yahoo.com.br 


\section{INTRODUÇÃO}

A importância do aleitamento materno para o desenvolvimento da criança em termos físicos e emocionais é incontestável, tanto que organizações nacionais e internacionais preocupam-se em estabelecer e difundir estratégias que incentivam e propiciam a amamentação.

Os estudos sobre o tema têm procurado demonstrar as práticas mais recomendáveis para a manutenção do aleitamento, principalmente o exclusivo, e sua contribuição na redução da morbi-mortalidade infantil. Nessa perspectiva, apontam-se intervenções com evidência científica de impacto positivo para o aumento das taxas de amamentação e para o seu retorno quando abandonada. Os meios indicados incluem aconselhamento individual, auxílio de agentes da comunidade, visitas domiciliares, suporte familiar e programas educativos durante o pré-natal e o puerpério(1-2).

Além disso, a estratégia Iniciativa Hospital Amigo da Criança (IHAC), difundida mundialmente, representa grande avanço no incentivo ao aleitamento exclusivo $^{(3-4)}$. A esse respeito, o que se verifica no Brasil é que a resistência de alguns profissionais arraigados a antigos conceitos e práticas obsoletas dificulta a expansão da IHAC.

Também, práticas relacionadas ao modelo de atenção adotado durante o processo de parto e nascimento podem estimular o aleitamento materno, na medida em que favorecem e proporcionam maior autonomia à mulher. Considera-se que parte dos problemas verificados na amamentação inexistiria caso posturas, atitudes e condutas mais humanizadas fossem utilizadas, destacando-se, entre elas, a participação ativa e orientada de acompanhantes no pré-natal e no parto e a restrição ao uso de intervenções desnecessárias e abusivas, como a cesárea eletiva ${ }^{(1)}$.

Nesse contexto, em atividade de promoção à saúde de mulheres de uma comunidade carente, desenvolvida pelas autoras, observa-se que nem todas as participantes foram assistidas no momento do parto em instituições que utilizam práticas humanizadas ou seguem as recomendações da IHAC. No acompanhamento dessas mulheres, realizado após o parto, verifica-se que muitas não referem o contato pele-a-pele com seus bebês e, nem mesmo, permanência em alojamento conjunto, a despeito das boas condições em que suas crianças nasceram.

Tendo em vista tais considerações, decidiu-se realizar pesquisa com o objetivo de verificar se a manutenção do aleitamento materno exclusivo nos primeiros seis meses de vida do bebê é influenciada pelas variáveis: contato pele-apele precoce e amamentação na primeira hora após o parto, permanência do binômio em alojamento conjunto, tipo de parto e tipo de hospital em que ocorreu o nascimento.

\section{MÉTODO}

\section{Tipo e Local da Pesquisa}

Pesquisa descritiva, exploratória e retrospectiva, com abordagem quantitativa, realizada no Núcleo São Lucas de Atendimento à Saúde da Mulher e da Criança, projeto de extensão e pesquisa que, desde o ano de 1999, é desenvolvido em uma comunidade carente do município de São Paulo. Nele, são colocados em prática pelos pesquisadores, normalmente acompanhados por estudantes ou bolsistas de graduação e de pós-graduação em Enfermagem, dois programas, um de Promoção à Saúde de Gestantes, no qual se realizam atividades educativas em grupos, e outro de Incentivo ao Aleitamento Exclusivo, em que as mulheres retornam com seus bebês após o parto e são acompanhadas por seis meses, em atendimentos individuais ou em grupo.

Este trabalho é semanalmente realizado em uma creche da favela de Paraisópolis, comunidade com cerca de 80 mil habitantes, situada na zona sul de São Paulo/SP e servida por apenas duas unidades básicas de saúde. Pela precariedade da comunidade é que algumas organizações sociais, entre elas o Núcleo, desenvolvem projetos com o intuito de complementar ou melhorar a atenção básica em saúde.

mais recomendáveis
para a manutenção do
aleitamento,
principalmente o
exclusivo, e sua
contribuição na
redução da morbi-
mortalidade infantil.

\section{Amostra}

Primeiramente foram analisadas as $120 \mathrm{fi}-$ chas de todas as mulheres que participaram dos grupos educativos e, depois, do acompanhamento pós-parto no período de fevereiro de 2003 a dezembro de 2006. Do total de documentos, foram selecionados os referentes às mães que compareceram de forma regular às consultas desde o primeiro retorno, geralmente na primeira quinzena após o parto, até o último, por volta do sexto mês de vida do bebê. Este foi o critério de inclusão que determinou coleta de dados em 75 fichas ou prontuários.

\section{Coleta dos Dados}

Foi realizada nas 75 fichas, nas quais constam informações obtidas por meio de entrevista e pela verificação de documentos referentes ao pré-natal, parto e pós-parto. Coletaram-se dados referentes à manutenção do aleitamento materno exclusivo nos primeiros seis meses de vida do bebê e das variáveis: contato pele-a-pele precoce e amamentação na primeira hora após o parto, permanência do binômio em alojamento conjunto, tipo de parto e tipo de hospital em que ocorreu o nascimento. Também, foram compiladas informações referentes à caracterização sócio-demográfica e obstétrica das mulheres, bem como intercorrências havidas com a mãe e o bebê após o nascimento.

\section{Análise dos Dados}

Para a correlação das variáveis em função do tempo de manutenção do aleitamento exclusivo nos primeiros seis 
meses de vida do bebê, foram determinados seis períodos de análise: de 0 a 30 dias, de 31 a 60 dias, de 61 a 90 dias, de 91 a 120 dias, de 121 a 150 dias e de 151 a 180 dias. Tendo em vista que as consultas nem sempre ocorriam no final desses períodos, foram utilizadas aproximações para menos, caso houvesse intervalos de anotações menores ou iguais a 15 dias, ou para mais, nos intervalos maiores que 15 dias.

Optou-se, também, por considerar unicamente aleitamento exclusivo (AE) e não exclusivo (ANE), devido à baixa incidência de aleitamento artificial em todos os primeiros períodos de análise. A classificação do tipo de aleitamento utilizada no Núcleo segue a definição da Organização Mundial da Saúde ${ }^{(3)}$, na qual exclusivo é o aleitamento em que a criança recebe somente leite do peito, diretamente da sua mãe, ou extraído, e nenhum outro líquido ou sólido, exceto gotas ou xaropes de vitaminas, suplementos vitamínicos ou medicamentos; misto é o aleitamento em que a criança recebe, além do leite materno, qualquer outro tipo de alimento ou líquido, como leite artificial, chás, sucos; artificial é a alimentação sem leite materno.

Na análise inferencial da influência das variáveis na manutenção do aleitamento nos seis períodos, foi utilizada a metodologia de Equações de Estimação Generalizada $(\mathrm{GEE})^{(5)}$, levando em conta a dependência entre os seis tempos de aleitamento e a distribuição binomial, AE ou ANE.

Foi feito um modelo para cada uma das variáveis, analisando-se a existência de efeito nos seis tempos, bem como os efeitos da interação entre cada variável e o tempo. Quando a interação não foi considerada estatisticamente significante, avaliaram-se o efeito de grupo, conjuntamente para todos os tempos, e o efeito de tempo, de forma conjunta para ambas as categorias de resposta da variável de interesse: contato precoce $(\mathrm{CP})$ ou contato não precoce $(\mathrm{CNP})$; alojamento conjunto ( $A C)$ ou alojamento não conjunto (ANC); parto normal (PN) ou parto operatório (PO), no qual foram incluídas as cesáreas e os fórcipes; hospital credenciado na IAHC (HAC) ou não credenciado (HNAC) na data de nascimento do bebê. Nas situações em que o efeito de interação foi significativo, as comparações entre os grupos foram feitas separadamente para cada tempo, assim como o efeito do tempo foi testado separadamente para cada categoria. Foram considerados estatisticamente significantes os resultados com $\mathrm{p}$-valores inferiores a 0,05.

\section{Procedimentos Éticos}

De acordo com a Resolução CONEP 196/96 que trata de pesquisas com seres humanos, todas as mulheres que aderem às atividades do Núcleo assinam Termo de Consentimento Livre e Esclarecido, em que constam os objetivos da organização, suas atividades de pesquisa, a participação livre, voluntária e consentida, a informação de que os dados do prontuário poderão ser utilizados para pesquisas e publicações, as garantias de sigilo, de identidade e de que não haverá riscos para sua pessoa ou a de seu filho em participar dos Programas. Por tratar-se de pesquisa a prontuários, não houve exposição dos sujeitos a riscos e agravos, sendo o estudo aprovado pelo Comitê de Ética da Universidade Guarulhos (Parecer no 150/2006).

\section{RESULTADOS}

Os dados referentes à caracterização sócio-demográfica e obstétrica e à assistência relatada no nascimento do último bebê, da Tabela 1, mostram maior freqüência de mulheres jovens, com idade entre 14 e 23 anos (53\%), que referem viver em união consensual com os companheiros (56\%), possuindo apenas o ensino fundamental (66\%), completo ou não; naturais e provenientes da região Norte-Nordeste do Brasil (56\%); sem emprego formal (89\%); que tiveram seu primeiro ou segundo filho (68\%); de parto normal (61\%); em hospitais não cadastrados na IHAC (85\%); sem contato pelea-pele ou amamentação na primeira hora após o parto (57\%) e com permanência da mãe e do recém-nascido (RN) em alojamento conjunto durante a internação (84\%).

Tabela 1 - Caracterização sócio-demográfica e obstétrica das mulheres - São Paulo - 2007

\begin{tabular}{|c|c|c|}
\hline CARACTERÍSTICAS & $N^{o}$ & $\%$ \\
\hline \multicolumn{3}{|l|}{ Idade } \\
\hline 14 a 18 anos & 14 & 19 \\
\hline 19 a 23 anos & 26 & 34 \\
\hline 24 a 28 anos & 14 & 19 \\
\hline 29 a 33 anos & 15 & 20 \\
\hline 34 anos ou mais & 6 & 8 \\
\hline \multicolumn{3}{|l|}{ Situação conjugal } \\
\hline União consensual & 42 & 56 \\
\hline Solteira & 17 & 23 \\
\hline Casada & 16 & 21 \\
\hline \multicolumn{3}{|l|}{ Escolaridade } \\
\hline Analfabeta & 2 & 3 \\
\hline Ensino fundamental incompleto & 42 & 55 \\
\hline Ensino fundamental completo & 8 & 11 \\
\hline Ensino médio incompleto & 8 & 11 \\
\hline Ensino médio completo & 15 & 20 \\
\hline \multicolumn{3}{|l|}{ Naturalidade } \\
\hline Região Norte e Nordeste & 42 & 56 \\
\hline Região Sul e Sudeste & 29 & 39 \\
\hline Região Centro-Oeste & 4 & 5 \\
\hline \multicolumn{3}{|l|}{ Situação empregatícia } \\
\hline Desempregada ou prendas domésticas & 67 & 89 \\
\hline Empregada e registrada & 8 & 11 \\
\hline \multicolumn{3}{|l|}{ Paridade } \\
\hline 1 a 2 & 51 & 68 \\
\hline 3 a 4 & 18 & 24 \\
\hline 5 a 6 & 5 & 7 \\
\hline 7 ou mais & 1 & 1 \\
\hline \multicolumn{3}{|l|}{ Tipo de parto (último) } \\
\hline Normal & 46 & 61 \\
\hline Cesárea & 24 & 32 \\
\hline Fórceps & 5 & 7 \\
\hline \multicolumn{3}{|l|}{ Local (último parto) } \\
\hline Hospital cadastrado na IHAC & 9 & 12 \\
\hline Hospital não cadastrado na IHAC & 64 & 85 \\
\hline Domicílio & 2 & 3 \\
\hline \multicolumn{3}{|l|}{$\begin{array}{l}\text { Aleitamento e/ou contato pele-a-pele na } \\
\text { primeira hora após último parto }\end{array}$} \\
\hline Sim & 32 & 43 \\
\hline Não & $43^{*}$ & 57 \\
\hline \multicolumn{3}{|l|}{$\begin{array}{l}\text { Permanência com o recém-nascido em } \\
\text { alojamento conjunto após último parto }\end{array}$} \\
\hline $\operatorname{Sim}$ & 63 & 84 \\
\hline Não & $12^{*}$ & 16 \\
\hline
\end{tabular}

* Oito casos de complicações com o RN que impediram o aleitamento e/ou contato pele-a-pele com a mãe e consequente permanência no alojamento conjunto. 
Quanto às justificativas para a não ocorrência de aleitamento e/ou contato pele-a-pele e para a separação do binômio no pós-parto e no alojamento conjunto, verificouse que em $8 \%$ dos casos elas se relacionaram a complicações que obrigaram o RN a permanecer em berçário por vários dias. No restante das vezes, não foi detectada qualquer explicação plausível para a separação.

Em relação à amamentação, observou-se que 92\% das mulheres iniciaram-na de forma exclusiva. Ao término do sexto mês de vida dos bebês, $31 \%$ deles permaneciam com AE e $62 \%$ com ANE.

No que concerne à variável contato precoce (CP ou CNP), sua correlação com o tipo de aleitamento mostrou que:

- Não existe efeito significativo da interação entre CP e tempo ( $p=0,4697)$, ou seja, o efeito da variável, se existir, não depende do tempo que está sendo analisado e o efeito de tempo, se existir, não depende do CP;

- Não existe efeito significativo do CP no tipo de aleitamento para todos os seis tempos de acompanhamento $(p=0,1746)$, isto é, o CP não interferiu na manutenção do $A E$;

- Existe efeito significativo para os grupos CP e CNP com relação ao tempo, ocorrendo diferença entre os tempos com relação ao tipo de aleitamento, pois o p-valor de cada relação foi sempre menor que 0,05 , ou seja, quanto mais avançado o tempo menor a chance de AE em ambos os grupos.

Este efeito pode ser visto na Figura 1, que mostra o percentual de $\mathrm{AE}$ nos seis primeiros meses segundo a variável contato precoce pele-a-pele e aleitamento na primeira hora após o parto.

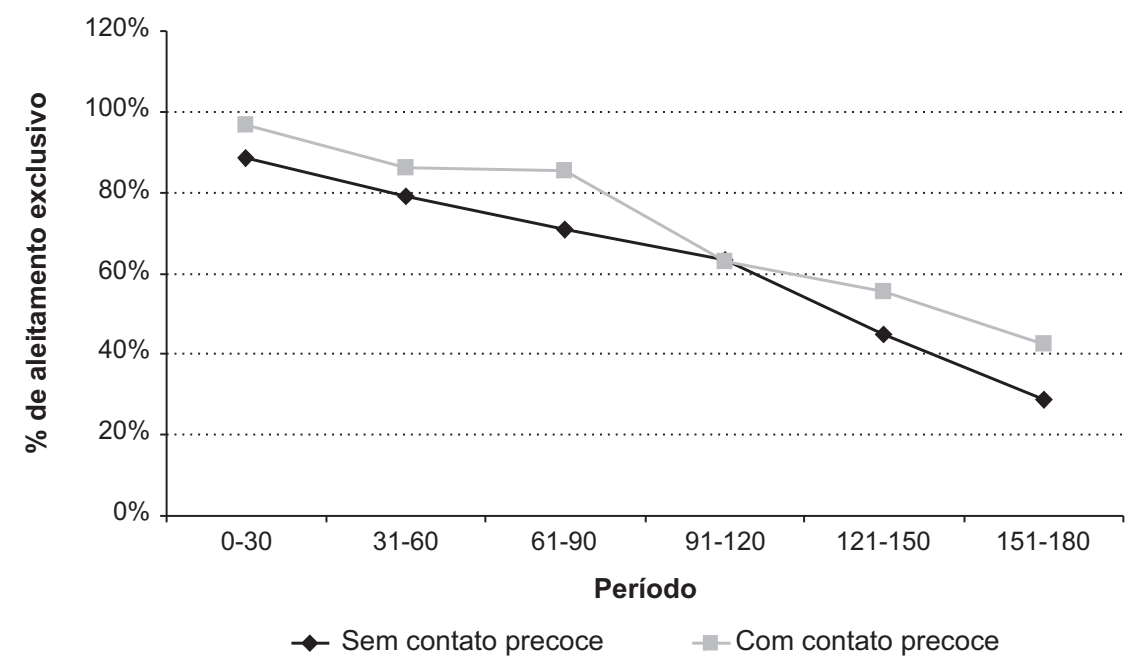

Figura 1 - Percentual de AE nos seis primeiros meses segundo tipo de contato - São Paulo - 2007

Em relação ao alojamento conjunto (AC e $A N C)$, sua correlação com o tipo de aleitamento mostrou que:

- Não existe efeito significativo da interação entre AC e tempo $(p=0,1032)$, ou seja, o efeito da variável, se existir, não depende do tempo que está sendo analisado e o efeito de tempo, se existir, não depende do AC;

- Existe efeito significativo de $\mathrm{AC}(p=0,0297)$ no tipo de aleitamento para todos os tempos de análise, isto é, AC interfere na manutenção do $\mathrm{AE}$;
- Existe efeito significativo para os grupos AC e ANC com relação ao tempo, havendo diferença entre os tempos com relação ao tipo de aleitamento, pois o p-valor de cada relação foi sempre menor que 0,05 . Observou-se que as mulheres em $A C$ têm 35 vezes mais chance de manter $A E$ quando comparadas com as em ANC em todos os tempos analisados, isto é, quanto mais avançado o tempo menor a chance de $A E$.

Este efeito pode ser visto na Figura 2, que mostra o percentual de $A E$ nos seis primeiros meses segundo a variável permanência da mãe em alojamento conjunto. 


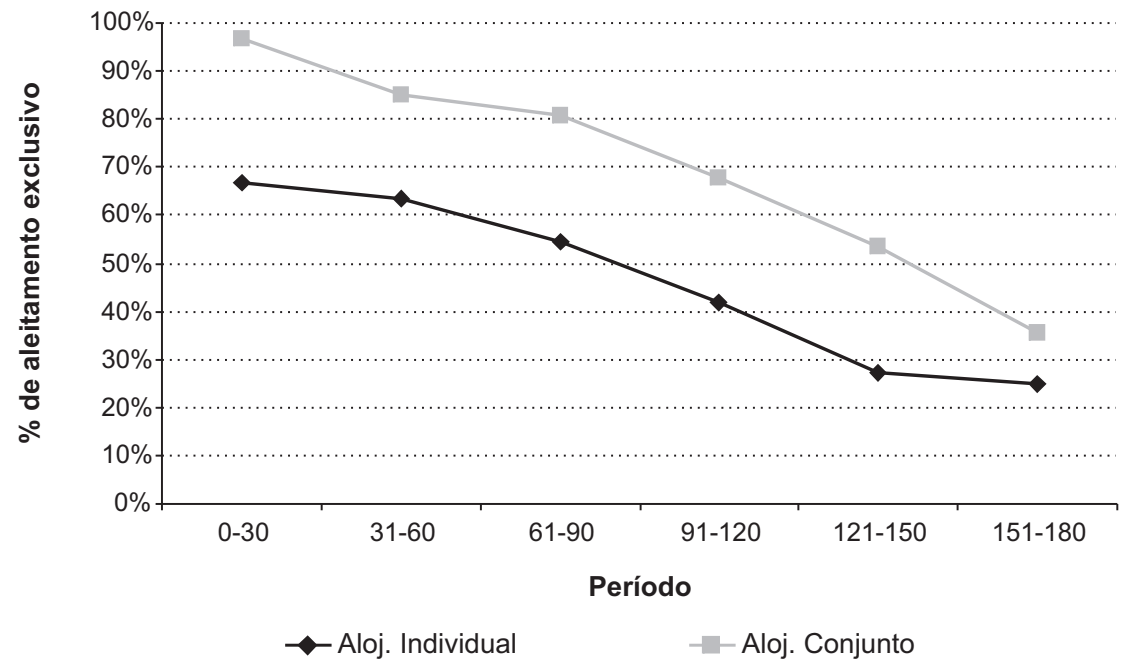

Figura 2 - Percentual de AE nos seis primeiros meses segundo tipo de alojamento - São Paulo - 2007

Quanto ao tipo de hospital (HAC e HNAC), na correlação desta variável com o tipo de aleitamento verificou-se que:

- Existe efeito significativo da interação entre HAC e tempo $(p=0,0001)$, ou seja, o efeito da variável, se existir, depende do tempo que está sendo analisado e o efeito de tempo, se existir, depende do HAC;

- Não existe efeito significativo de HAC $(p=0,4308)$ no tipo de aleitamento para todos os tempos de análise, isto é, $\mathrm{HAC}$ não interfere na manutenção do $\mathrm{AE}$;
- Não existe efeito significativo para o grupo HAC com relação ao tempo, pois todos os $p$-valores são superiores a 0,05 ;

- Existe efeito significativo para o grupo HNAC com relação ao tempo, com todos os p-valores inferiores a 0,05 , havendo diferença entre os tempos com relação ao tipo de aleitamento, ou seja, quanto mais avançado o tempo menor a chance de AE no grupo HNAC.

Este efeito pode ser visto na Figura 3, que mostra o percentual de $A E$ nos seis primeiros meses segundo o tipo de hospital.

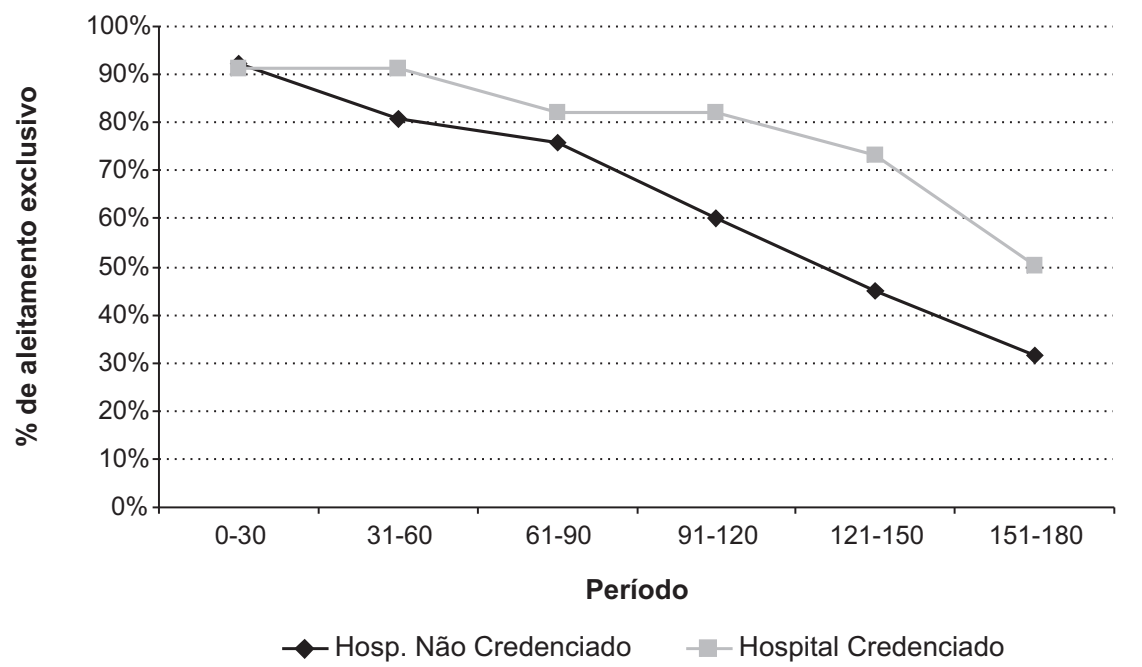

Figura 3 - Percentual de AE nos seis primeiros meses segundo credenciamento do hospital à IHAC - São Paulo - 2007

No que se refere à última variável, tipo de parto (PN ou PO), a correlação entre ela e o tipo de aleitamento mostrou que:

- Existe efeito significativo da interação entre o tipo de parto e o tempo ( $p=0,0001)$, ou seja, o efeito da variável, se existir, depende do tempo que está sendo analisado e o efeito de tempo, se existir, depende do tipo de parto;

- Comparando os tipos de parto com o tipo de aleitamento materno, houve diferença significativa $(p=0,0389)$, ape- nas no 10 período (0-30 dias), no qual as mulheres que tiveram PN apresentam maior chance de manter AE quando comparadas às que se submeteram a PO. Nos outros períodos não ocorreu diferença estatisticamente significante $(p>0,05)$;

- Para as mulheres com PN, há diferença significativa na comparação de todos os tempos $(p<0,05)$, com exceção do 3ㅇ período com o 2 으 $(p=0,1552)$ e do 50 período com o 4 ㅇ $(p=0,0792)$, observando-se que quanto maior o período menor a chance de ocorrer $\mathrm{AE}$; 
- Para as mulheres com PO, também houve diferença significativa na comparação de todos os tempos $(p<0,05)$, com exceção do $2 \circ$ período com o $10(p=0,3092)$ e do 4 으 período com o $30(p=0,5645)$, ou seja, quanto maior o período menor a chance de AE.
Estas diferenças podem ser vistas na Figura 4, que mostra o percentual de AE nos seis primeiros meses segundo o tipo de parto.

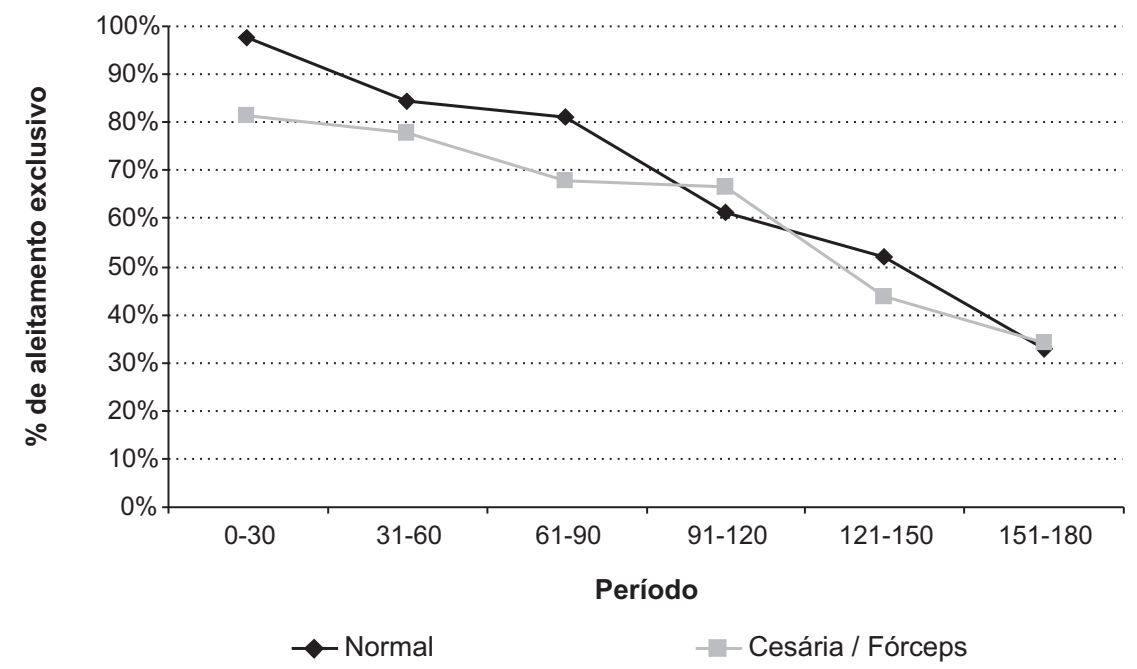

Figura 4 - Percentual de AE nos seis primeiros meses segundo tipo de parto - São Paulo - 2007

\section{DISCUSSÃO}

Destacam-se, entre os dados de caracterização, por interferirem negativamente na instalação e manutenção do aleitamento materno ${ }^{(2,4,6-8)}$, a baixa escolaridade e alto índice de mães adolescentes, fatores que alertam para a possibilidade de ocorrência de agravos à saúde do bebê, pois as principais causas da morbi-mortalidade no primeiro ano de vida estão intimamente ligadas às condições de saúde e nutrição das crianças, ao nível de escolaridade e de vida das mães, bem como à qualidade da atenção no ciclo gravídico-puerperal ${ }^{(9)}$.

A despeito de essas mulheres terem recebido informações sobre amamentação durante a gestação, outros fatores podem ter interferido na diminuição de sua autoconfiança para amamentar como, por exemplo, a separação.

Quando se pensa na separação de mães e bebês logo após o parto, supõe-se a existência de intercorrências à saúde materna ou neonatal. Esta, no entanto, não é a realidade encontrada, pois na grande maioria dos casos não foram relatados ou documentados problemas que explicassem a falta de contato e de aleitamento na primeira hora pós-parto, mesmo em hospitais já cadastrados na IHAC, o que é inexplicável e vai contra a promoção da amamentação.

Mesmo não havendo diferença significativa do ponto de vista estatístico que pudesse comprovar a interferência do $C P$ na manutenção do $A E$, foram evidenciados maiores índices desse tipo de amamentação em, praticamente, todos os seis períodos de acompanhamento.

As evidências mostram que o CP aumenta o aleitamento materno, tanto imediatamente após o parto quanto dois ou três meses mais tarde, além de promover efeitos no comportamento materno e auxiliar o estabelecimento de

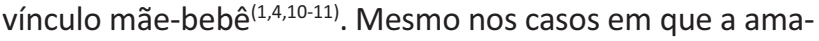
mentação é contra-indicada, apenas o contato pele-a-pele deve ser encorajado, não havendo qualquer motivo que justifique os altos índices de separação logo após o parto, verificados neste estudo.

Tanto o CP quanto a permanência em $\mathrm{AC}$, quarto e sétimo passos da IHAC, favorecem não só o aleitamento materno como também o estabelecimento do vínculo mãefilho, com conseqüente diminuição dos casos de abandono do bebê pela mãe(12).

Outro empecilho ao aleitamento, infelizmente ainda presente na cidade de São Paulo, tanto em hospitais privados quanto nos conveniados ao SUS, é a separação mãebebê no pós-parto. Nesta situação, em que os recém-nascidos permanecem em berçários até o momento da alta hospitalar, questiona-se como as mães podem ganhar confiança para amamentar se não lhes é permitido, ao menos, estar continuamente ao lado de seu bebê, aprendendo a conhecê-lo e a cuidá-lo.

O AC propicia, também, oportunidades de aconselhamento ou de ajuda prática durante as mamadas, de forma que a mãe se sinta mais segura para lidar com as mais vari- 
adas pressões para que não amamente ou que ofereça alimentos complementares ao recém-nascido(13).

A análise estatística mostrou que a ocorrência do AC interfere a favor do aparecimento de maiores índices de $A E$, ou seja, provou-se a interferência dessa variável na manutenção da $A E$.

Além de outros aspectos, destaca-se que o AC estimula a mãe a amamentar sob livre demanda, sem restrições quanto à freqüência e duração das mamadas, o que pode impedir também a utilização de chupetas, com todos os malefícios que podem acarretar ${ }^{(1,4)}$. Mesmo quando mãe e bebê são separados, pode haver sucesso neste processo(4), especialmente quando a mulher acredita na sua capacidade de amamentar, encontra suporte e desenvolve segurança no pós-parto, trabalho desenvolvido continuamente por todos os que atuam no Núcleo.

Os dados de aleitamento, mesmo limitados pela pequena amostra que compõe este estudo, denotam resultados animadores, ou seja, $72 \%$ das mães acompanhadas amamentaram seus bebês por pelo menos seis meses. O desmame, quando ocorreu, somente atingiu patamares superiores a $10 \%$ a partir dos três meses, em média aos 108 dias.

Como já ressaltado, estas taxas não são representativas da realidade brasileira, cuja mediana de AE é de 23 dias ${ }^{(11)}$. De qualquer forma, acredita-se que investir na promoção da amamentação, sem imposições e de forma acolhedora, é tarefa social de relevância, tendo em vista as circunstâncias em que as mulheres vivem e criam seus filhos naquela comunidade carente ${ }^{(2)}$.

Diversos estudos relacionam o aleitamento materno, mesmo do tipo misto, com a redução da diarréia e das doenças respiratórias, principais causas de internação hospitalar entre menores de um ano no Estado de São Paulo. Esses trabalhos enfatizam também que programas de promoção do aleitamento, como a IAHC, resultam em maior tempo de amamentação, o dobro até da mediana nacional ${ }^{(7,13-14)}$.

Portanto, a IHAC repercute positivamente nas taxas de aleitamento materno, fato evidenciado neste estudo, na medida em que foi comprovado efeito significativo entre HAC e $A E$ em, praticamente, todos os períodos do acompanhamento. $\mathrm{O}$ fracasso em tornar as maternidades amigas da criança não pode ser defensável, bem como a implementação de apenas alguns dos passos da IHAC. As evidências mostram que os dez passos devem ser praticados em conjunto, o que exige apoio continuado às mães, bem como capacitação e treinamento de todos os profissionais da instituição, mesmo aqueles não envolvidos diretamente na assistência ${ }^{(4)}$.

A análise estatística mostrou também efeito significativo da interação entre PN e AE, especialmente no primeiro mês de vida do bebê. Partos cirúrgicos acarretam maiores complicações à saúde materna e perinatal, sendo normalmente relacionados às dificuldades no início da amamentação, especialmente nos hospitais não credenciados à IHAC ${ }^{(15)}$.
Mães com parto operatório também têm maior dificuldade em aleitar, devido ao mau posicionamento ou ao inadequado envolvimento afetivo. Além disso, a cesárea retarda ou dificulta as primeiras mamadas por alterar as respostas endócrinas da mãe e do recém-nascido logo após o parto, indicando que o ato cirúrgico provoca dor e sonolência, e o uso de anestésicos e analgésicos afeta a interação mãe/bebê $\hat{e}^{(15)}$.

Todos esses fatores contribuem para a diminuição do aleitamento materno e conseqüente oferta de líquidos complementares aos neonatos, o que resulta em menores índices de amamentação, especialmente no primeiro mês de vida $^{(12,15-17)}$, resultado também encontrado neste trabalho.

\section{CONCLUSÕES}

Conclui-se que o tipo de hospital, a permanência em alojamento conjunto e o tipo de parto influenciaram a manutenção do AE durante os seis primeiros meses de vida dos bebês desta pesquisa.

Em relação ao contato precoce, quarto passo da IAHC favorecedor não só do aleitamento como também do vínculo mãe-filho, não se constatou sua influência na manutenção da amamentação exclusiva.

Mostrou-se que os índices de $\mathrm{AE}$ foram maiores nos casos em que mãe e bebê permaneceram constantemente juntos após o parto, quando os nascimentos aconteceram em hospitais amigos da criança e após partos normais. Constatou-se, assim, que, além de outros aspectos, a assistência recebida pela mulher durante o processo de parto e nascimento influencia a amamentação.

Tais resultados mostram o quanto ainda se tem que fazer para melhorar a assistência materno-infantil em nosso país, tanto na atenção à mulher quanto na implantação e manutenção das estratégias que compõem a IHAC, além de outras políticas de incentivo ao aleitamento.

Por ser o aleitamento um ato cultural, social e político de múltiplas e amplas dimensões, muitos profissionais de saúde se encontram despreparados para com ele lidar. Além disso, os modelos assistenciais ainda vigentes na atenção obstétrica e neonatal não propiciam apoio à mulher no processo de amamentar no momento do parto ou, até mesmo, depois.

É importante frisar que a extensão da duração da amamentação não depende de estratégias isoladas, mas sim da combinação de diferentes intervenções colocadas em prática em todo o ciclo gravídico-puerperal. Os dados deste trabalho mostram a efetividade dos programas do Núcleo na promoção do aleitamento materno, especialmente no apoio regular e no suporte às nutrizes, além das estratégias que aumentam o conhecimento das mulheres durante a gestação. 
Todas essas práticas devem fazer parte do cotidiano dos profissionais que investem na promoção do aleitamento materno, especialmente em relação às famílias carentes, para as quais suas vantagens são evidentes. Os índices encontrados estimulam a manutenção e aprimoramento dos programas do Núcleo na medida em que auxiliam a supe-

\section{REFERÊNCIAS}

1. Carvalho MR, Tamez RN. Amamentação: bases científicas. 2aa. ed. Rio de Janeiro: Guanabara Koogan; 2005.

2. Narchi NZ, Fernandes RAQ, Gomes MMF, Queiroz ML, Higasa DN. Análise da efetividade de um programa de incentivo ao aleitamento exclusivo em comunidade carente na cidade de São Paulo. Rev Bras Saúde Mater Infant. 2005;5(1):87-92.

3. Organização Mundial da Saúde (OMS). Evidências científicas dos dez passos para o sucesso no aleitamento materno. Brasília; 2001.

4. Toma TS, Monteiro CA. Avaliação da promoção do aleitamento materno nas maternidades públicas e privadas do município de São Paulo. Rev Saúde Pública. 2001;35(5):409-14.

5. Twisk JWR. Applied longitudinal data analysis for epidemiology. Cambridge: Cambridge University Press; 2003.

6. Lima TM, Osório MM. Perfil e fatores associados ao aleitamento materno em crianças menores de 25 meses na Região Nordeste do Brasil. Rev Bras Saúde Matern Infant. 2003;3(3):305-14.

7. Venâncio SI, Escuder MML, Kitoko P. Freqüência e determinantes do aleitamento materno em municípios do Estado de São Paulo. Rev Saúde Pública. 2002;36(3):313-8.

8. Ramos CV, Almeida JAG. Aleitamento materno: como é vivenciado por mulheres assistidas em uma unidade de saúde de referência na atenção materno-infantil em Teresina, Piauí. Rev Bras Saúde Matern Infant. 2003;3(3):315-21.

9. Brasil. Ministério da Saúde. Secretaria de Vigilância em Saúde. Saúde Brasil 2004: uma análise da situação de saúde. Brasília; 2004. rar os efeitos adversos decorrentes da inadequada atenção materno-infantil. Investir nessa temática é tarefa social de relevância, mormente quando se leva em conta o contexto adverso da comunidade em que vivem as mulheres deste estudo e suas famílias.

10. Bueno LGS, Teruya KM. Aconselhamento em amamentação e sua prática. J Pediatr. 2004;80 Supl:126-30.

11. Brasil. Ministério da Saúde. Secretaria de Políticas de Saúde da Criança. Prevalência de aleitamento materno nas capitais brasileiras e no Distrito Federal. Brasília; 2001.

12. Klaus M. Mother and infant: early emotional ties. Pediatrics. 1998;102(5 Suppl E):1244-6.

13. Escuder MML, Venâncio SI, Pereira JC. Estimativa de impacto da amamentação sobre a mortalidade infantil. Rev Saúde Pública. 2003;37(3):319-25.

14. São Paulo (Estado) Secretaria de Estado da Saúde. Coordenadoria de Controle de Doenças. Mortalidade infantil no Estado de São Paulo: diagnóstico de situação e propostas de intervenção para redução dos indicadores de mortalidade materna e infantil. São Paulo: SES; 2005.

15. Carvalhaes MABL, Corrêa CRH. Identificação de dificuldades no início do aleitamento materno mediante aplicação de protocolo. J Pediatr. 2003;79(1):13-20.

16. Kummer SC, Giugliani ERJ, Susin LO, Folletto JL, Lermen NR, Wu VYJ, et al. Evolução do padrão de aleitamento materno. Rev Saúde Pública. 2000;34(2):143-8.

17. Aragaki IMM, Silva IA, Santos JLF. Traço e estado de ansiedade de nutrizes com indicadores de hipogalactia e nutrizes com galactia normal. Rev Esc Enferm USP. 2006;40(3):396-403. 